Psychological Medicine, 2000, 30, 997-1003. Printed in the United Kingdom

(C) 2000 Cambridge University Press

EDITORIAL

\title{
Vascular dementia as a frontal subcortical system dysfunction ${ }^{1}$
}

Vascular dementia (VaD) is the second most common subtype of dementia in Western countries (Desmond, 1996) and, overall, may be the most common subtype of dementia in the world (Henderson, 1994). Furthermore, the recognition of some major risk factors of cerebrovascular disease makes VaD a form of 'preventable senility' (Hachinski, 1992). The last decade has seen a major re-evaluation of the concept of VaD (Erkinjuntti \& Hachinski, 1993; Hachinski, 1994), with new diagnostic criteria having been proposed (World Health Organization, 1993; American Psychiatric Association, 1994) but without any consensus (Wetterling et al. 1996). Indeed, some investigators have called for the abandonment of the diagnosis of $\mathrm{VaD}$ and the adoption of alternative nosology (Hachinski, 1994). It is therefore time to re-examine the concept of VaD and evaluate its distinctive features.

\section{THE EVOLUTION OF THE CONCEPT OF VASCULAR DEMENTIA}

The origins of the concept of $\mathrm{VaD}$ can be traced to Binswanger (1894), who proposed the condition 'encephalitis subchronicalis chronica progressiva' considered to arise from pathology in deep perforating cerebral vessels that resulted in the formation of lacunae of infarction and demyelination of deep white matter with the preservation of cortical grey matter. It emerged that Binswanger's disease was a rare cause of $\mathrm{VaD}$ (Lishman, 1987) and it was then subsumed under the rubric of arteriosclerotic dementia. Arteriosclerotic dementia was postulated to arise from ischaemic cerebral injury due to arteriosclerotic narrowing of the cerebral vasculature (Loeb \& Meyer, 1996). Tomlinson and colleagues (1970) distinguished Alzheimer's disease (AD) from VaD, and demonstrated the importance of cerebral infarction for $\mathrm{VaD}$. Following the observation that large vessel pathology contributed to the majority of $\mathrm{VaD}$ cases, the term multi-infarct dementia (MID) was proposed by Hachinski et al. (1974) to describe dementia associated with multiple cerebral infarcts of varying size. For nearly two decades, MID became synonymous with VaD. Following re-evaluation in the 1990s, the concept of $\mathrm{VaD}$ was broadened to include cognitive impairment caused by cerebrovascular disease in its various forms. It was recognized that brain impairment could be caused by non-infarcting vascular lesions, which could vary in their distribution, pathology and aetiology. The stage for a reconceptualization of $\mathrm{VaD}$ had been set.

\section{WHICH DEFINITION OF VaD SHOULD BE ADOPTED?}

The two major features of $\mathrm{VaD}$ are the presence of sufficient cognitive impairment to qualify for the diagnosis of dementia, and the demonstration of cerebrovascular disease as an aetiological factor. Recently, $\mathrm{VaD}$ has been conceptualized as a heterogeneous syndrome resulting from a number of differing vascular pathological processes and mechanisms, including: embolism, thrombosis, small vessel disease, lacunae etc. (McPherson \& Cummings, 1996; Sachdev et al. 1999). There has also been a focus on white matter changes as aetiological factors for VaD. Attempts to define VaD have

\footnotetext{
1 Address for correspondence: Dr Jeffrey C. L. Looi, NPI, McNevin-Dickson Building, Prince of Wales Hospital, High Street, Randwick, NSW 2031, Australia.
} 
involved characterization of the patterns of dysfunction and the study of radiological and pathological material. Neuropsychological testing has been utilized to assist in the differentiation of $\mathrm{VaD}$ and $\mathrm{AD}$ by characterization of the cognitive deficits in each disorder.

The term $\mathrm{VaD}$ has been adopted by major research groups and diagnostic systems. The proposed set of diagnostic criteria can be classified into two broad groups (Bowler \& Hachinski, 1997): those formulated as part of a series of general diagnostic criteria for psychiatric disorders (ICD-10, World Health Organization, 1992; DSM-IV, American Psychiatric Association, 1994); and those developed specifically as operationalized research criteria (Alzheimer's Diseases Diagnostic and Treatment Centers (ADDTC), Chul et al. 1992; National Institute of Neurological Disorders and Stroke-Association pour la Recherché et l'Ensignment en Neurosciences (NINDS-AIREN) Roman et al. 1993). While classification systems are not necessarily intrinsically valid, they nonetheless shape our views of clinical syndromes through their use in research and clinical practice. Therefore, the current systems need to be critically examined.

With the exception of the ADDTC criteria, memory impairment is required as an essential feature in all major proposals. This emphasis on memory impairment reflects a definition of dementia that has been shaped by the characteristic features of AD. Whether memory impairment should be considered an essential feature of $\mathrm{VaD}$ has not been empirically established. We argue in this paper that the focal disturbance in $\mathrm{VaD}$ may well be in another cognitive domain. This is not a solely academic exercise as there are consequences of the application of traditional 'dementia' concept to $\mathrm{VaD}$. First, patients are likely to be diagnosed at a relatively advanced stage of their illness with $\mathrm{VaD}$, precluding preventative measures (Bowler \& Hachinski, 1997). Secondly, functional disturbance in $\mathrm{VaD}$ is complicated by stroke-related physical impairment which usually does not directly relate to neuropsychological dysfunction of $\mathrm{VaD}$. The criteria require us to distinguish between the two, which is difficult in the clinical situation. Thirdly, VaD, as currently defined, may develop as a result of multiple infarcts, non-infarct vascular disease, single strategic infarcts, lacunar infarcts, haemorrhage, etc., and the clinical profiles of these 'subtypes' can vary considerably. Should one then have a single construct of VaD? It has been suggested that the term VaD may have lost its usefulness and should be abandoned (Hachinski, 1994), perhaps to be replaced with the term Vascular Cognitive Impairment (VCI). Is this the way forward?

We address this issue by examining the question whether $\mathrm{VaD}$, as currently defined, and $\mathrm{AD}$ do have different neuropsychological profiles. We also review the neuroimaging literature to determine the possible neuroanatomical basis of these neuropsychological deficits. What is the 'core' neuropsychological disturbance in patients diagnosed as having $\mathrm{VaD}$, and can this form the basis for a new model of $\mathrm{VaD}$ that more accurately describes patients with $\mathrm{VaD}$ ?

\section{NEUROPSYCHOLOGICAL DIFFERENTIATION OF VAD FROM AD}

In determining a valid definition of $\mathrm{VaD}$, it is inevitable that a comparison be drawn with $\mathrm{AD}$. Almkvist (1994) published a qualitative review of neuropsychological deficits in $\mathrm{VaD}$ and compared them with those deficits found in $\mathrm{AD}$. He concluded that $\mathrm{VaD}$ patients, when compared to $\mathrm{AD}$ of similar severity, had greater deficits in frontal-executive functions, verbal fluency, attention and motor function. The author also noted a relative advantage for $\mathrm{VaD}$ compared to $\mathrm{AD}$ in naming and intrusion errors. He reported that verbal long-term memory was relatively preserved in VaD. For many other cognitive functions, there was either little difference in performance between the dementia groups or the functions had been inadequately assessed in the published literature. $\mathrm{He}$ ascribed the differences in performance to differences in pathology between $\mathrm{VaD}$ and $\mathrm{AD}$ suggesting that: (i) VaD may be characterized by lesions in subcortical and sensorimotor projection areas; and (ii) AD may be characterized by lesions in the limbic and posterior cortical areas, with relative sparing of sensorimotor areas. The conclusion was that frontal-subcortical dysfunction was more pronounced in $\mathrm{VaD}$ than $\mathrm{AD}$. He also noted methodological problems in the studies reviewed: the use of clinical as opposed to population samples, failure to match adequately, and the relative paucity of studies attempting to correlate structural and functional changes. 
Bowler \& Hachinski (1997) concluded group differences on neuropsychological testing between $\mathrm{VaD}$ and $\mathrm{AD}$ were negligible. Multiple possible aetiologies for $\mathrm{VaD}$ could each feature a characteristic pattern of neuropsychological deficits (McPherson \& Cummings, 1996).

We recently completed a systematic literature review of the utility of neuropsychological test performance in differentiating VaD from AD (Looi \& Sachdev, 1999). This review highlighted two main areas of differential neuropsychological test performance between the two groups: verbal learning and memory; and, executive function. Other domains of neuropsychological test performance were either insufficiently investigated or unhelpful in distinguishing between the dementia groups. Our review supported Almkvist's (1994) conclusions in suggesting that frontal-executive dysfunction and not memory impairment, was the most prominent feature of $\mathrm{VaD}$. A potential criticism of any such analysis is that of circularity of argument, since the findings are likely to be influenced by the selection criteria adopted by a study. In the case of $\mathrm{VaD}$, the bias is likely to be in minimizing the differences from $\mathrm{AD}$ in neuropsychological function. The reported differences, as summarized above are therefore unlikely to be an exaggeration, even though further work is necessary that utilizes novel ways of diagnosing $\mathrm{VaD}$.

\section{STRUCTURAL-FUNCTIONAL CORRELATIONS FOR FRONTAL EXECUTIVE DYSFUNCTION}

Further support for these differential performances in neuropsychological function was derived from the examination of structural-functional correlations between neuroimaging and neuropsychological test performance in studies that were included in our review (Looi \& Sachdev, 1999). Starkstein et al. (1996) performed both MRI and SPECT imaging on $10 \mathrm{VaD}$ and $20 \mathrm{AD}$ patients. The SPECT scans demonstrated significantly lower blood flow in the frontal regions, specifically, the frontal cortex and frontal basal ganglia of those with VaD. Group differences were demonstrated in the form of increased levels of both white matter and infratentorial hyperintensities on MRI in $\mathrm{VaD}$. They concluded that deficits seen in $\mathrm{VaD}$ patients in planning, set alteration, verbal fluency, anosognosia and pathological crying suggested disruption of frontal-subcortical circuits. Villardita (1993) performed MRI scans, and noted that 16 of the $30 \mathrm{VaD}$ patients had frontal lesions. He compared these 16 patients with 14 non-frontal $\mathrm{VaD}, 48 \mathrm{AD}$ and 48 normal controls. Both $\mathrm{AD}$ and frontal $\mathrm{VaD}$ patients performed better than non-frontal $\mathrm{VaD}$ patients on the complex figure test. It was also noted that frontal $\mathrm{VaD}$ patients were more compromised than non-frontal $\mathrm{VaD}$ and $\mathrm{AD}$ subjects on the Porteus maze and visual pattern completion. This study again supported the correlation of frontal-subcortical cognitive deficits with frontal lesions. Lafosse et al. (1997) investigated the number of infarcts, white matter lucency, ventricular enlargement and cortical atrophy on CT scans in $20 \mathrm{VaD}$ patients. They reported that more severe leukoaraiosis was associated with poorer verbal fluency and Memory Assessment Scale (MAS) performance. An increasing number of infarcts was related to poorer performance on the MAS. Greater cortical atrophy was associated with poorer performance on all neuropsychological tests except those for verbal fluency. Libon et al. (1997) investigated primarily subcortical pathology (i.e. periventricular and deep white matter alterations), measuring deep white matter alterations using a leukoaraiosis score. They found that a more severe level of leukoaraiosis was associated with poorer performance on tests of executive function (Weschler Memory Scale mental control) but superior performance on memory tests (CVLT-D). They proposed that the differences in cognitive function between AD and subcortical $\mathrm{VaD}$ subjects were analogous to those of other subcortical dementias.

Recent neuropathological studies have shed further light on the contrasting pathology of both $\mathrm{VaD}$ and $\mathrm{AD}$. In a neuropathological series of 18 elderly non-demented subjects free of cerebrovascular disease, 19 elderly non-demented subjects with cerebrovascular disease and 24 elderly demented subjects with cerebrovascular disease, Esiri et al. (1997) reported that microvascular brain damage (severe cribriform change) and associated subcortical white matter damage and micro-infarction were correlated with a history of dementia, whereas macroscopic infarction was not. Functional imaging utilizing positron emission tomography (PET) has 
demonstrated that two possible mechanisms may underlie $\mathrm{VaD}$ : chronic cerebral ischaemia in patients with lacunar and subcortical damage and reduced cortical blood flow in patients with multiple large infarcts (De Reuck et al. 1998). Furthermore, Jellinger (1998) observed that multiple cortical infarctions are much less frequent causes of cognitive impairment in cerebrovascular disease than multiple subcortical lesions (lacunes and subcortical encephalopathy) secondary to microvascular disease and hypertension. Thus, this subcortical white matter pathology in VaD may form the substrate for frontal-subcortical dysfunction. In contrast, atrophy of the amygdala (Cuenod et al. 1993) and hippocampus (Jack et al. 1991) have been described in AD suggesting differential pathology from $\mathrm{VaD}$. The neuropsychological correlates of such atrophy would indicate temporal-mnestic dysfunction.

The neurochemical changes of $\mathrm{VaD}$ have been examined through the utilization of magnetic resonance spectroscopy (MRS). In a study using phosphate ${ }^{31} \mathrm{P}-\mathrm{MRS}$, ten patients with multiple subcortical infarct dementia were compared with 17 patients with AD and 17 normal controls (Brown et al. 1989). Elevated phophocreatinine to inorganic orthophosphate levels were found in the temporoparietal and frontal regions in the vascular dementia subjects, suggesting altered phospholipid metabolism, increased neuronal component synthesis or altered cortical energy metabolism in these regions (Brown et al. 1989). Also utilizing ${ }^{31} \mathrm{P}-\mathrm{MRS}$ a subsequent study found increased phosphate energy charge in cerebral cortical areas, especially frontally, in 18 vascular dementia patients with multiple subcortical ischaemic lesions (Brown et al. 1993). This increased charge has been hypothesized to arise from either metabolic hypoactivity or gliosis associated with cortical ischaemia (Brown et al. 1993). Five patients with subcortical dementia and extensive white matter hyperintensities (WMH) were compared with four asymptomatic subjects with WMH and five elderly controls in one study utilizing proton MRS (Brooks et al. 1997). The $\mathrm{N}$-acetylaspartate (NAA) to creatinine (Cr) and NAA to choline (Cho) ratios were reduced in the five demented subjects when compared with the asymptomatic groups. In comparison with controls, the NAA was decreased and Cho elevated. These neurochemical changes were consistent with neuronal injury such as chronic demyelination or ischaemia in those with subcortical dementia (Brooks et al. 1997). A larger study of eight patients with subcortical ischaemic vascular dementia, 40 patients with AD and 18 elderly controls found lower NAA/Cr ratios in those with vascular dementia (MacKay et al. 1996). The NAA/Cr ratio was significantly lower in frontal white matter voxels in vascular dementia subjects compared with controls. The MRS findings support the hypothesis of differential neurochemical dysfunction in both frontal and subcortical systems in VaD compared with AD.

\section{COMMON NEUROPATHOLOGICAL CHANGES IN AD AND VaD}

However, there may be a subgroup of dementia patients in which overlap of $\mathrm{AD}$ and $\mathrm{VaD}$ neuropathology occurs, resulting in dementia. Scheltens et al. (1992) found that more white matter lesions were found in later onset $\mathrm{AD}$, this group showing more MRI white matter changes than was normal for age. Similarly, a greater extent of periventricular hyperintensities (PVH) has been observed in AD patients in comparison with controls, even after matching for cerebrovascular risk factors (Fazekas et al. 1996), but it was suggested that the extent of PVH was a proxy for brain atrophy rather than an independent finding. Snowdon et al. (1997) found at autopsy, in those who met neuropathological criteria for $\mathrm{AD}$, those with brain infarction had poorer cognition and higher prevalence of dementia. Those with lacunar infarctions in the basal ganglia, thalamus or deep white matter had an especially high prevalence of dementia. Fewer neuropathological lesions of AD were required to result in dementia in those with lacunar infarcts in these regions than those without. Snowdon et al. (1997) concluded that cerebrovascular disease may therefore play a role in the expression of $\mathrm{AD}$. Therefore, there is some evidence to suggest interaction between the neuropathology of $\mathrm{AD}$ and $\mathrm{VaD}$, possibly leading to a greater proportion of mixed dementias than previously postulated. 


\section{THE FRONTAL-SUBCORTICAL CIRCUITS AND FRONTAL DYSFUNCTION}

The frontal impairments observed in $\mathrm{VaD}$ have been hypothesized as resulting from dysfunction in frontal-subcortical circuits (Cummings, 1994). These circuits typically consist of neuronal connections from frontal cortex to basal ganglia to thalamus with feedback from the thalamus to the frontal cortex (Alexander et al. 1986). The parallel neuronal circuits of particular relevance to our discussion are the dorsolateral prefrontal, the orbito-frontal and the anterior cingulate circuits (Alexander et al. 1986). The dorsolateral prefrontal circuit (DLPFC) subserves frontal executive function, and strokes in this region often manifest neuropsychiatrically as depression and anxiety (Mega \& Cummings, 1994). The lateral orbitofrontal circuit (OFC) typically manifests dysfunction as personality change: irritability, lability, tactlessness, fatuous euphoria (Mega \& Cummings, 1994) and damage to the ventromedial frontal cortex, causing deficits in real-life decision-making (Bechara et al. 1994) may manifest as acquired sociopathy (Saver \& Damasio, 1991). The anterior cingulate circuit (ACC) mediates motivational behaviour and lesions manifest as apathy (Mega \& Cummings, 1994). The circuits potentially implicated in VaD include the DLPFC mediating executive function; OFC mediating emotional lability and the ACC responsible for motivation and initiation (Cummings, 1993, 1994). Thus, frontal-subcortical circuits may serve as models to help conceptualize executive dysfunction in VaD.

\section{A NEW PARADIGM FOR VASCULAR DEMENTIA}

Given the findings on the relative preservation of verbal learning and memory in VaD compared to $\mathrm{AD}$, the emphasis on memory impairment in the conceptualization of $\mathrm{VaD}$ is questionable. The requirement for memory impairment in existing conceptualizations of $\mathrm{VaD}$ may bias the samples of VaD towards those that closely resemble AD (Bowler \& Hachinski, 1997). This may explain some of the current difficulties in distinguishing between the dementia types on neuropsychological testing.

In the current definitions of $\mathrm{VaD}$, frontal-executive dysfunction is not a necessary criterion for the diagnosis. Since it is a common and important aspect of $\mathrm{VaD}$ and distinguishes it from $\mathrm{AD}$ as much as can be currently ascertained, greater importance should be assigned to this feature. We suggest that this neuropsychological dysfunction, based on disruptions of frontal-subcortical circuits, may be the salient disturbance in $\mathrm{VaD}$. The pathology does not have to be exclusively in the white matter; the involvement of subcortical grey matter and the frontal cortex would have similar consequences. Since cerebrovascular disease is not restricted to these brain regions, heterogeneous neuropsychological deficits may be superimposed on this frontal-subcortical disturbance. However, in the absence of the latter, cerebrovascular disease produces patchy impairment that would not qualify for status as a discrete syndrome even though some criteria for dementia may be met. This may explain the difficulties that many physicians have in diagnosing dementia in patients with one or more strokes.

That subcortical pathology due to vascular factors may lead to a dementia syndrome has been recognized for over a century (Binswanger, 1894), but whether all $\mathrm{VaD}$ patients have significant subcortical pathology has not been investigated. It may seem that we wish to turn the concept of $\mathrm{VaD}$ almost full circle to Binswanger's model of subcortical pathology. This would not be entirely accurate. First, Binswanger focused on the white matter, whereas we would extend the concept to the subcortical nuclei and even the cortex. Secondly, the cases described by Binswanger (1894) were too incompletely characterized to be able to attribute any definite neuropathology to them (Pantoni $\&$ Garcia, 1995). Thirdly, Binswanger described normal cortical grey matter which is not necessary in this model. Fourthly, the model of subcortical pathology has been augmented with the conceptualization of frontal-subcortical circuit dysfunction.

This conceptualization of $\mathrm{VaD}$ has implications for clinical practice and research. Sensitivity to frontal-executive dysfunction should inform the diagnosis of VaD. Patients with early and 
significant memory impairment, when they also have evidence for cerebrovascular disease affecting frontal-subcortical circuits, will be more readily identified as possibly having 'mixed' dementia. Research in $\mathrm{VaD}$ will develop a clearer focus in trying to understand the mechanisms of frontal-subcortical dysfunction as a substrate for $\mathrm{VaD}$. We suggest that future research should heed this model of VaD.

\section{JEFFREY C. L. LOOI AND PERMINDER S. SACHDEV}

Dr J. C. L. Looi was supported by a NSW Institute of Psychiatry Research Fellowship and a project grant from the National Health and Medical Research Council of Australia. We wish to thank Michael Valenzuela for references on MRS and Professor Gordon Parker for editorial comments.

\section{REFERENCES}

Alexander, G. E., Delong, M. R. \& Strick, P. L. (1986). Parallel organisation of functionally segregated circuits linking basal ganglia and cortex. Annual Review of Neuroscience 9, 357-381.

Almkvist, O. (1994). Neuropsychological aspects of vascular dementia in relation to Alzheimer's disease: reviewing the evidence for functional similarity or divergence. Dementia 5, 203-206.

American Psychiatric Association (1994). Diagnostic and Statistical Manual of Mental Disorders, Fourth Edition (DSM-IV), pp. 143-147. American Psychiatric Association: Washington, DC.

Bechara, A., Damasio, A. R., Damasio, H. \& Anderson, S. W. (1994). Insensitivity to future consequences following damage to human prefrontal cortex. Cognition 50, 7-15.

Binswanger, O. (1894). Die Abrenzung der allegmeinen progresiven Paralyse. Berliner Kilnische Wochenschrift 13, 1137-1139.

Bowler, J. V. \& Hachinski, V. (1997). Vascular dementia. In Behavioural Neurology and Neuropsychology (ed. T. E. Feinberg and M. J. Farah), pp. 589-603. McGraw-Hill: New York.

Brooks, W. M., Wesley, M. H., Kodituwakku, P. W., Garry, P. J. \& Rosenberg, G. A. (1997). ${ }^{1} \mathrm{H}-\mathrm{MRS}$ differentiates white matter hyperintensities in subcortical arteriosclerotic encephalopathy from those in normal elderly. Stroke 28, 1940-1943.

Brown, G. G., Levine, S. R., Gorell, J. M., Pettegrew, J. W., Gdowski J. W., Bueri J. A., Halpern, J. A. \& Welch, K. M. A. (1989). In vivo 31P profiles of Alzheimer's disease and multiple subcortical infarct dementia. Neurology 39, 1423-1427.

Brown, G. G., Garcia, J. H., Gdowski, J. W., Levine, S. R. \& Helpern, J. A. (1993). Altered brain energy metabolism in demented patients with multiple subcortical ischemic lesions. Archives of Neurology 50, 384-388.

Chui, H. C., Victoroff, J. I., Margolin, D., Jagust, W., Shankle, R. \& Katzman, R. (1992). Criteria for the diagnosis of ischemic vascular dementia proposed by the state of California Alzheimer's Disease Diagnostic and Treatment Centers. Neurology 42, 473-480.

Cuenod, C. A., Denys, A., Michot, J. L., Jehenson, P., Forette, F., Kaplan, D., Syrota, A. \& Boller, F. (1993). Amygdala atrophy in Alzheimer's disease. Archives of Neurology 50, 941-945.

Cummings, J. L. (1993). Frontal subcortical circuits and human behaviour. Archives of Neurology 5, 873-880.

Cummings, J. L. (1994). Vascular subcortical dementias: clinical aspects. Dementia 5, 177-180.

De Reuck, J., Decoo, D., Marchau, M, Santens, P., Lemahieu, I. \& Strijckmans, K. (1998). Positron emission tomography in vascular dementia. Journal of Neurological Sciences 154, 55-61.

Desmond, D. W. (1996). Vascular dementia: a construct in evolution. Cerbrovascular and Brain Metabolism Review 8, 296-325.

Erkinjuntti, T. \& Hachinski, V. C. (1993). Rethinking vascular dementia. Cerebrovascular Disorders 3, 3-23.

Esiri, M. M., Wilcock, G. K. \& Morris, J. H. (1997). Neuropathological assessment of the lesions of significance in vascular dementia. Journal of Neurology, Neurosurgery and Psychiatry 63, 749-753.

Fazekas, F., Kapeller, P., Schmidt, R, Offenbacher, H., Payer, F. \& Fazekas, G. (1996). The relation of cerebral magnetic resonance signal hyperintensities to Alzheimer's disease. Journal of the Neurological Sciences 142, 121-125.

Hachinski, V. (1992). Preventable senility: a call for action against the vascular dementias. Lancet 340, 645-648.

Hachinski, V. (1994). Vascular dementia: a radical redefinition. Dementia 5, 130-132.

Hachinski, V., Lassen, N. A. \& Marshall, J. (1974). Multi-infarct dementia. Lancet ii, 207-209.

Henderson, A. S. (1994). Dementia. World Health Organization: Geneva.

Jack, C. R., Peterson, R. C., O'Brien, P. C. \& Tanglos, E. G. (1991). MRI-based hippocampal volumetry in the diagnosis of Alzheimer's disease. Neurology 42, 183-188.

Jellinger, K. A. (1998). Positron emission tomography in vascular dementia. Journal of the Neurological Sciences 160, 190-191.

Lafosse, J. M., Reed, B. R., Mungas, D., Sterling, S. B., Wahbeh, H. \& Jagust, W. J. (1997). Fluency and memory differences between ischaemic vascular dementia and Alzheimer's disease. Neuropsychology 11, 514-522.

Lishman, W. A. (1987). Organic Psychiatry (2nd edn), pp. 385-390. Blackwell Scientific: Oxford.

Loeb, C. \& Meyer, J. S. (1996). Vascular dementia: still a debatable entity? Journal of Neurological Sciences 143, 31-40.

Looi, J. C. L. \& Sachdev, P. S. (1999). The differentiation of vascular dementia from Alzheimer's disease on neuropsychological test performance. Neurology 52, 670-678.

MacKay, S., Meyerhoff, D. J., Constans, J. M., Norman, D., Fen, G \& Weiner, M. W. (1996). Regional gray and white matter metabolite differences in subjects with $\mathrm{AD}$, with subcortical ischaemic vascular dementia and elderly controls with ${ }^{1} \mathrm{H}$ magnetic resonance spectroscopic imaging. Archives of Neurology 53, 167-174

McPherson, S. E. \& Cummings, J. L. (1996). Neuropsychological aspects of vascular dementia. Brain and Cognition 31, 261-282.

Mega, M. S. \& Cummings, J. L. (1994). Frontal-subcortical circuits and neuropsychiatric disorders. Journal of Neuropsychiatry and Clinical Neurosciences 6, 358-370.

Pantoni, L. \& Garcia, J. H. (1995). The significance of cerebral white matter abnormalities 100 years after Binswanger's report: a review. Stroke 26, 1293-1301

Roman, G. C., Tatemichi, T. K., Erkinjuntti, T., Cummings, J. L., Masdeu, J. C., Garcia, J. H. et al. (1993). Vascular dementia: diagnostic criteria for research studies: report of the NINDSAIREN International Workshop. Neurology 43, 250-260.

Sachdev, P. S., Brodaty, H. \& Looi, J. C. L. (1999). Vascular dementia: diagnosis, management and prevention. Medical Journal of Australia 170, 81-85.

Saver, J. L. \& Damasio, A. R. (1991). Preserved access and processing of social knowledge in a patient with acquired sociopathy due to ventromedial frontal damage. Neuropsychologia 29, 124-129.

Scheltens, P., Barkhof, F., Valk, J., Algra, P. R., van der Hoop, R. G., Nauta, J. \& Wolters, E. C. (1992). White matter lesions on magnetic resonance imaging in clinically diagnosed Alzheimer's disease. Brain 115, 735-748.

Snowdon, D. A., Greiner, L. H., Mortimer, J. A., Riley, K. P., Greiner, P. A. \& Markesbery, W. R. (1997). Brain infarction and 
clinical expression of Alzheimer disease: the nun study. Journal of the American Medical Association 277, 813-817.

Starkstein, S. E., Sabe, L., Vasquez, S., Teson, A., Petracca, G., Chemerinski, E., Di Lorenzo, G. \& Leguarda, R. (1996). Neuropsychological, psychiatric and cerebral blood flow findings in vascular dementia and Alzheimer's disease. Stroke 27, 408-414.

Tomlinson, B. E., Blessed, G. \& Roth, M. (1970). Observations on the brains of demented old people. Journal of Neurological Science 11, 205-242.
Villardita, C. (1993). Alzheimer's disease compared with cerebrovascular dementia: neuropsychological similarities and differences. Acta Neurologica Scandanavica 87, 299-308.

Wetterling, T., Kanitz, R. D. \& Borgis, K. J. (1996). Comparison of different diagnostic criteria for vascular dementia (ADDTC, DSMIV, ICD-10, NINDS-AIREN). Stroke 27, 30-36.

World Health Organization (1993). The ICD-10 Classification of Mental and Behavioural Disorders: Diagnostic Criteria for Research pp. 36-40. World Health Organization: Geneva. 